\title{
A differential equation for specific catchment area
}

\author{
John C. Gallant ${ }^{1}$ and Michael F. Hutchinson ${ }^{2}$ \\ Received 20 August 2009; revised 21 February 2011; accepted 28 February 2011; published 25 May 2011. \\ [1] Analysis of the behavior of specific catchment area in a stream tube leads to a simple \\ nonlinear differential equation describing the rate of change of specific catchment area \\ along a flow path. The differential equation can be integrated numerically along a \\ flow path to calculate specific catchment area at any point on a digital elevation model \\ without requiring the usual estimates of catchment area and width. The method is \\ more computationally intensive than most grid-based methods for calculating specific \\ catchment area, so its main application is as a reference against which conventional \\ methods can be tested. This is the first method that provides a benchmark for more \\ approximate methods in complex terrain with both convergent and divergent areas, not just \\ on simple surfaces for which analytical solutions are known. Preliminary evaluation of \\ the D8, M8, digital elevation model networks (DEMON), and D $\infty$ methods indicate that \\ the $\mathrm{D} \infty$ method is the best of those methods for estimating specific catchment area, but \\ all methods overestimate in divergent terrain.
}

Citation: Gallant, J. C., and M. F. Hutchinson (2011), A differential equation for specific catchment area, Water Resour. Res., 47, W05535, doi:10.1029/2009WR008540.

\section{Introduction}

[2] Specific catchment area $a$ is one of the most commonly used hydrological terrain attributes. On its own it can be used as a surrogate for discharge per unit flow width, and it is commonly used in combination with slope $S$ as either the topographic wetness index TWI $=\ln (a / S)$ [Beven and Kirkby, 1979; Quinn et al., 1991, 1995] or as the stream power index $\omega=a S$ [Moore and Burch, 1986; Moore and Wilson, 1992]. These indices have many applications in predicting patterns of saturation [Beven and Kirkby, 1979; Güntner et al., 2004], saturated subsurface flow [Mishra et al., 2008], channel initiation [Dietrich et al., 1993; McNamara et al., 2006], erosive power [Moore and Wilson, 1992], and soil properties [McKenzie et al., 2000; Gessler et al., 1995] and as indices of topographic position for mapping landforms [Ventura and Irvin, 2000] and vegetation distribution [Mackey et al., 2000].

[3] In spite of its wide use and a general consensus on its meaning, specific catchment area does not seem to have been clearly defined, perhaps because there has been no need for the precision of a definition in the applications to date. The mathematical treatment presented in this paper provides a motivation for a clear definition and examination of the assumptions implicit in the definition.

[4] We define specific catchment area $a$ at a point as

$$
a=\lim _{w \rightarrow 0} \frac{A}{w},
$$

\footnotetext{
${ }^{1}$ CSIRO Land and Water, Canberra, ACT, Australia.

${ }^{2}$ Fenner School of Environment and Society, Australian National University, Canberra, ACT, Australia.
}

Copyright 2011 by the American Geophysical Union. 0043-1397/11/2009WR008540 with $A$ being the area of land surface, as vertically projected onto the horizontal plane, between two slope lines that originate at a common hilltop, bounded at the lower end by a contour segment of length $w$ as shown in Figure 1. While, in general, the upper end of area $A$ can be bounded by multiple hilltops and drainage divides, we restrict attention in our analysis to the case of a single hilltop. As noted by Maxwell [1870], each point in the land surface can be essentially allocated in this way to a unique hilltop. Points on slope lines originating from saddle points are at the conjunction of slope lines from two neighboring hilltops and require special treatment. Area $A$ is called the contributing or catchment area or sometimes total catchment area to distinguish it from specific catchment area [Gruber and Peckham, 2009]. An equivalent definition is

$$
a=\frac{\partial A}{\partial k},
$$

where $k$ is distance along the contour line from an arbitrary starting point; this will be explored with more rigor later in the paper.

[5] This definition is stated in terms of slope lines [Cayley, 1859], or lines of slope [Maxwell, 1870], that follow the direction of steepest descent across the land surface rather than flow lines that follow the direction of water flow. This is in keeping with the use of specific catchment area as a topographic attribute with hydrological and other applications. The connection between specific catchment area and surface water flow is strong wherever water flow is determined primarily by the slope gradient but breaks down where other factors, such as momentum and pressure gradients, become significant. These other factors are important in concentrated flow such as channels and in areas that are flat, or very nearly so, but are usually insignificant for diffuse surface flow and shallow subsurface flow on hillslopes. 


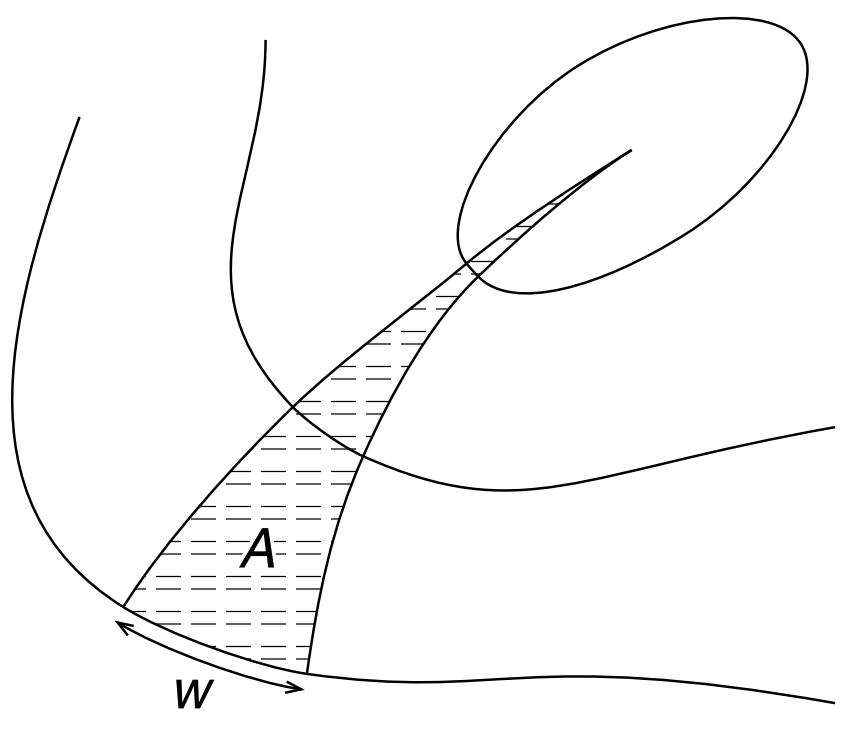

Figure 1. An idealized stream tube originating at a hilltop and terminating at a contour on a hillslope. The average specific catchment area $a$ along the contour segment is the ratio of contributing area $A$ to flow width $w$.

Specific catchment area is therefore of value as a hydrological index on hillslopes but less so in nearly flat areas and channels.

[6] The definition assumes the existence of slope lines following the direction of steepest descent that vary in a continuous fashion along well-defined elevation contours. This implies, at the minimum, a continuous land surface with continuous first derivatives of elevation and nonzero slope. This is, of course, violated at cliffs and overhangs, in flat areas, and at sharp ridges and valleys where slope lines with different origins can coalesce. However, away from such areas it is reasonable to assume a high degree of surface continuity, and it is in these areas where specific catchment area is a useful measure. Indeed, the main result of this paper is couched in terms of an integral of second derivatives, so it is convenient to assume for this analysis that the land surface has piecewise continuous second derivatives. This is consistent with the use of biquadratic splines to interpolate across a regular grid digital elevation model (DEM), as adopted in section 6 .

[7] Calculating specific catchment area from regular grid DEMs remains a challenge, as witnessed by the number of algorithms that have been developed since the early 1980s: (1) D8 steepest descent single downslope flow direction [O'Callaghan and Mark, 1984]; (2) Rho8 randomized version of D8, designed to reduce the parallel flow path artifacts of D8 [Fairfield and Leymarie, 1991]; (3) slopeweighted multiple downslope direction methods [Freeman, 1991; Quinn et al., 1991, 1995], collectively called M8 here; (4) digital elevation model networks (DEMON) flow tube method [Costa-Cabral and Burges, 1994], based on the aspect driven method of Lea [1992]; (5) Do continuous single-flow direction with flow proportioned among adjacent neighboring grid cells [Tarboton, 1997]; (6) D8-LTD and D8-LAD, modified D8 methods that reduce the deviation between the ideal flow path and the single-flow direction path [Orlandini et al., 2003]; and (7) MD $\infty$, a slope-weighted multiple-direction modification of Tarboton's D $\infty$ method [Seibert and McGlynn, 2007].

[8] The diversity of methods have arisen in response to the difficulty in reconciling the curved paths taken by water flowing across complex topography with the rectilinear structure of a grid DEM. Flow paths diverge and converge in different parts of the landscape and are rarely aligned with the orientation of the grid cells. The different methods encompass a range of compromises between simplicity and accuracy.

[9] The deficiencies in the two simplest methods, D8 and M8, have been clearly identified and discussed by CostaCabral and Burges [1994] and Tarboton [1997]. In summary, the D8 method cannot disperse flow in divergent terrain, cannot follow aspect angles that are not multiples of $45^{\circ}$, and has significant errors for noncardinal flow directions, while the M8 method produces excessive dispersion and introduces dependencies between cells that contradict actual flow directions. The modified D8-LTD method [Orlandini et al., 2003] substantially improves the representation of flow paths while retaining the nondispersive nature of the D8 method. The DEMON method [CostaCabral and Burges, 1994] is one of the more accurate methods because it explicitly traces flow paths, but this comes at a high computational cost. The D $\infty$ method [Tarboton, 1997] also appears to be quite accurate and is computationally efficient. Both DEMON and D $\infty$ methods are still effectively single-flow direction algorithms at the scale of a single grid cell, so neither is well adapted to strongly divergent terrain. The M8 and MD $\infty$ methods can disperse flow to several adjacent grid cells in strongly diverging terrain such as hilltops and sharp ridges.

[10] Catchment areas and flow widths can also be determined from contour data if slope lines between contours can be robustly determined. The methods of Dawes and Short [1994], building upon previous work by O'Loughlin [1986] and Moore and Grayson [1991], provided a reasonably robust approach, but Moretti and Orlandini [2008] demonstrated the first comprehensive solution to this problem. One key to the success of Moretti and Orlandini's approach was the construction of additional skeleton lines that capture the ridges and valleys implied by the curvature of the contour lines. This parallels the contour interpolation method of Hutchinson [1988] that employs automatically derived curvilinear ridge lines and streamlines to address this issue, as described by Hutchinson and Gallant [2000]. Having constructed a network of slope lines and contour lines, catchment areas can be defined for each contour segment and specific catchment area calculated using (1) without the limit due to the finite width.

[11] All existing methods, whether working from grids or from contours, compute specific catchment area by calculating $A$ and $w$ separately and then calculating a mean or effective $a$ as the ratio in (1) without the limit. A number of different methods have been proposed for determining $w$ on a grid, generally without a sound theoretical basis [Chirico et al., 2005]. Errors in the estimation of both $A$ and $w$ will contribute to errors in estimates of $a$.

[12] Evaluating the accuracy of specific catchment area algorithms requires knowledge of exact $a$, or $A$ and $w$, so that the accuracy of the method can be established. To date, 


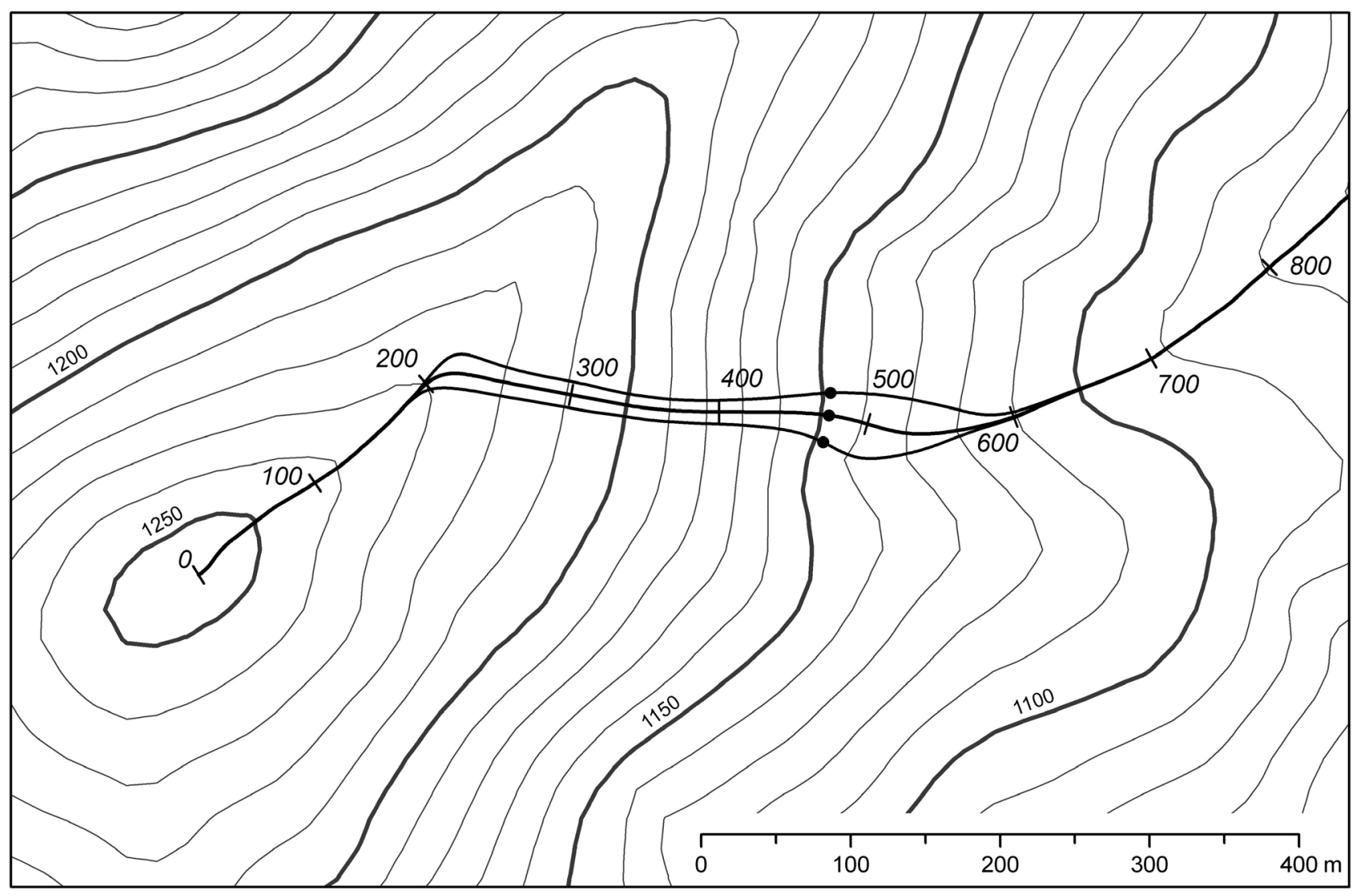

Figure 2. A section of the Brindabella $20 \mathrm{~m}$ resolution DEM showing contours at $10 \mathrm{~m}$ intervals and slope lines forming a stream tube flowing from a hilltop on the left to a valley on the right. The labeled tick marks along the central slope line show distance from the hilltop in meters. The starting points for construction of the lines are shown as dots on the three lines.

these comparisons have used simple mathematical surfaces, namely, convergent and divergent cones and inclined planes, where $a$ can be determined from first principles. The accuracy of the methods on complex surfaces cannot be established, and the usual approach is to make comparisons between methods coupled with an intuitive expectation of what the results should look like. The one exception to this is the detailed comparison by Orlandini and Moretti [2009] of surface flow paths and catchment areas from several gridbased methods using a detailed contour and flow line network [Moretti and Orlandini, 2008] as a reference. That study was conducted in steep terrain where the contourbased network provided a good representation of the surface flow pathways. It showed that mildly dispersive methods such as $\mathrm{D} \infty$ provided better spatial patterns of contributing area than nondispersive methods, particularly in divergent terrain, but the path-based nondispersive methods such as D8-LTD provided a better representation of flow paths across the surface.

[13] This paper derives a differential equation for $a$ that can be solved numerically to yield specific catchment area at any point on any smooth surface. The equation yields $a$ directly without requiring separate calculation of $A$ and $w$. This approach does not at this stage provide an efficient method for calculating specific catchment area for a whole DEM, but it does provide for the first time an analytical benchmark against which other methods can be tested on complex surfaces. The software and data used in this paper can be obtained from the first author.

\section{Specific Catchment Area Derived From a Stream Tube}

[14] A stream tube is the tract of land defined by two adjacent slope lines that delivers water to a channel [Onstad and Brakensiek, 1968]. A third slope line can be chosen between the two lines defining the stream tube, along which specific catchment area can be estimated. It should be noted that this slope line can only be an approximation to the "center" of the flow tube since the horizontal separations between different slope lines can vary as they descend down the hillslope. At any point along this third slope line, a contour line can be constructed to the edges of the stream tube, and specific catchment area can be calculated as $a=A / w$ using the area $A$ above the contour line and length $w$ of the contour line.

[15] Figure 2 shows such a stream tube derived from a $20 \mathrm{~m}$ resolution DEM of a section of the Brindabella ranges bordering the Australian Capital Territory in southeastern Australia $\left(35^{\circ} 21^{\prime} \mathrm{S}, 148^{\circ} 49^{\prime} \mathrm{E}\right)$ [Moore et al., 1993]. The DEM was produced by the ANUDEM program [Hutchinson, $1988,1989,2006]$ from 1:25,000 scale contours and streamlines. This landscape is dominated by steep slopes with narrow ridges and valleys. A starting point on a hillslope was 


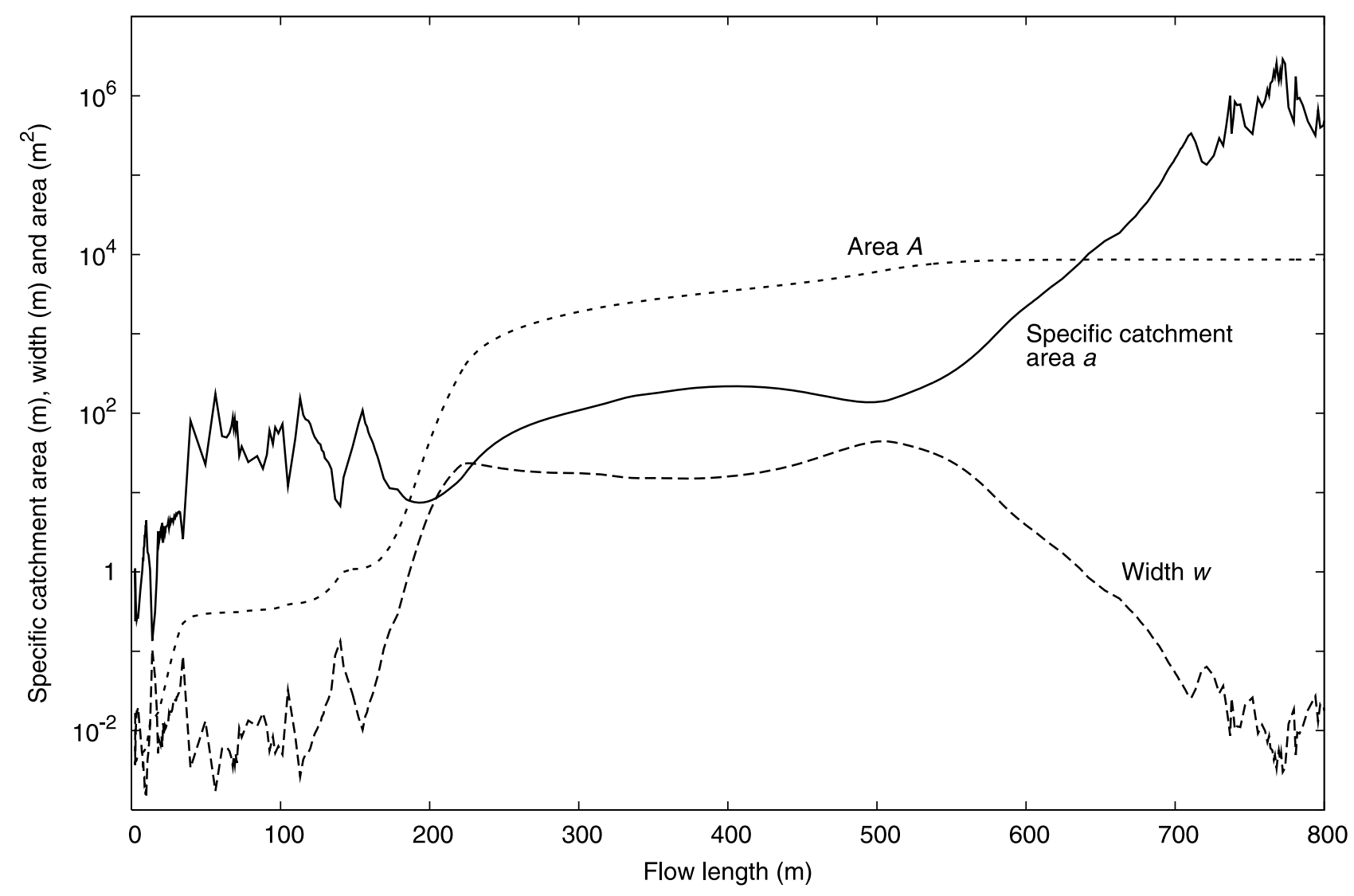

Figure 3. Flow width (or contour length) $w$, contributing area $A$, and specific catchment area $a$ computed from the stream tube of Figure 2. The flow width is not reliably estimated in the strongly divergent ridgeline $(0-190 \mathrm{~m})$ or strongly convergent valley $(>640 \mathrm{~m})$.

chosen for the "third" line, and two points about $20 \mathrm{~m}$ to either side of the first point define the starting points for the two lines defining the stream tube. Lines were constructed (using methods described in section 5) upslope from the starting points until they reached the hilltop, then the lines were extended downward until they converged within the valley. For each point on the third line, the elevation was calculated and points were identified on the left and right line at the same elevation. The contour segment was approximated as straight lines between the third line and the two side lines, from which $w$ is readily calculated. The quadrilateral elements between successive contour lines on either side of the third line define incremental areas that are accumulated from the top of the slope lines to determine contributing area $A$.

[16] Figure 3 shows the width $w$, contributing area $A$, and specific catchment area $a$ for the stream tube as a function of flow length down the hill. The point chosen to anchor the slope lines is located on the nearly planar hillslope at a flow length of $473 \mathrm{~m}$ from the hilltop. This method estimates $a$ effectively where the width is sufficiently large but not where the width becomes very small. The construction of slope lines by numerical integration of flow direction (aspect) causes an accumulation of positional error in the slope lines as they progress further upslope or downslope from the starting point. Eventually, the errors in position cause the constructed lines to cross over each other and the stream tube width loses accuracy (this is a purely numerical artifact: the actual slope lines of course cannot cross or merge as long as first derivatives are continuous). In this example, the width is considered to be sufficiently accurate while it remains above $1 \mathrm{~m}$. Note that "accurate" here is meant to imply not an accurate representation of flow lines on the real surface but an accurate construction of slope lines on the smooth surface defined by the DEM; see section 5 for further discussion on this point.

[17] In the portion where width is greater than $1 \mathrm{~m}$ (from about 190 to $640 \mathrm{~m}$ flow length), the specific catchment area increases, decreases slightly, and then increases again. These three segments correspond to (1) the divergent (190$220 \mathrm{~m}$ ), then approximately planar section of the hillslope with constant stream tube width $(220-400 \mathrm{~m})$; (2) the mildly divergent section where the stream tube broadens (400$500 \mathrm{~m}$ ); and (3) the converging section $(500-640 \mathrm{~m})$. In the first section specific catchment area is almost constant in the short divergent part, where $w$ increases from about 2 to $20 \mathrm{~m}$, then increases approximately linearly (appearing as a curved line in Figure 3 because of the logarithmic scale) in the planar part because of linearly increasing $A$ and approximately constant $w$. In the second section the area continues to increase at an increasing rate, but the increasing width results in a decrease of specific catchment area. Beyond $500 \mathrm{~m}$ the decreasing width results in a continuously increasing $a$ even though the rate of increase in area reduces and the area is essentially constant beyond $600 \mathrm{~m}$.

[18] The problems with inaccurate line positions could be treated by more sophisticated numerical methods or by taking explicit measures to prevent the lines from crossing. 


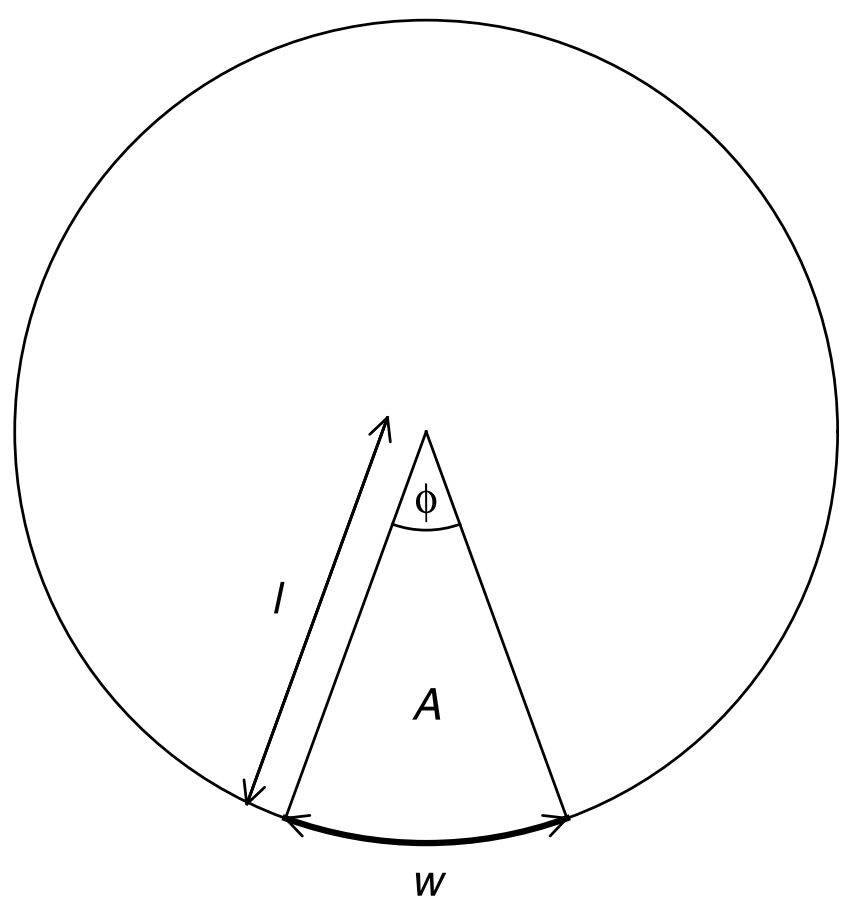

Figure 4. A stream tube on a conical hilltop. The radius of curvature $r_{c}$ of the contour is equal to the flow path length $l$.

Other complications with this approach arise when the slope lines constructed upslope do not terminate on the same hilltop, which is a problem that must be addressed by any method that relies on explicit construction of slope lines [Dawes and Short, 1994; Moretti and Orlandini, 2008]. The definition of width and the location of the "middle" of the stream tube can also be difficult in more complicated cases. Fortunately, all these difficulties with the construction of stream tubes can be avoided by looking more closely at the behavior of specific catchment area along a single slope line.

\section{Derivation of the Main Result}

[19] Here we show that the relationships between area $A$ and width $w$ can be used to derive specific catchment area $a$ along a slope line without separately determining $A$ and $w$.

[20] In the first instance, consider a stream tube on a conical surface where the slope lines are straight lines originating at the peak of the cone and contours are circles as shown in Figure 4. The area of the stream tube $A$ at distance $l$ from the hilltop is the integral of flow width $w$ with respect to flow length:

$$
A(l)=\int_{0}^{l} w(t) d t
$$

or in differential form

$$
\frac{d A}{d l}=w .
$$

On the cone, the width $w$ increases linearly along the flow path

$$
w=l \phi,
$$

where $\phi$ is the angle between the slope lines at the peak. The differential form is

$$
\begin{gathered}
\frac{d w}{d l}=\phi, \\
=\frac{w}{l}, \\
=w \kappa_{c},
\end{gathered}
$$

where $\kappa_{c}$ is the curvature of the contour line, equal to the inverse of the radius of curvature $r_{c}$, which in this case is the flow path length $l$. Here we adopt the convention that contour curvature is positive for divergent flow paths as shown in Figure 4.

[21] From the definition of specific catchment area

$$
\begin{gathered}
\frac{d a}{d l}=\frac{d}{d l}\left(\lim _{w \rightarrow 0} \frac{A}{w}\right), \\
=\lim _{w \rightarrow 0} \frac{w \frac{d A}{d l}-A \frac{d w}{d l}}{w^{2}}, \\
=\lim _{w \rightarrow 0} \frac{w \cdot w-A w \kappa_{c}}{w^{2}}, \\
=1-\lim _{w \rightarrow 0} \frac{A}{w} \kappa_{c}, \\
=1-a \kappa_{c} .
\end{gathered}
$$

This equation provides an explicit differential equation describing the evolution of specific catchment area along a slope line in this simple case and demonstrates a direct link between specific catchment area and contour curvature, also known as plan curvature.

[22] To address the general case, we use an orthogonal curvilinear coordinate system that naturally corresponds with the surface slope and contour lines. Figure 5 shows the coordinate system with $u$ as the coordinate that varies along the contour and $v$ as the coordinate that varies along the slope line. We define the orientations of $u$ and $v$ so that $v$ increases in the downhill direction and $u$ increases to the right when facing downhill, producing a right-handed coordinate system.

[23] The mathematical aspects of curvilinear coordinate transformations are described in some detail by Courant [1937, volume 2, section 3.3] and Jeffreys and Jeffreys [1956, sections 4.14 and 5.052], and the relevant elements are stated here. The $(u, v)$ used here correspond to Courant's $(\xi, \eta)$ and Jeffreys and Jeffreys' $\left(x^{\prime 1}, x^{\prime 2}\right)$.

[24] The transformation from $(u, v)$ to $(x, y)$ coordinates is represented by functions

$$
x=g(u, v), \quad y=h(u, v) .
$$

There is a corresponding inverse transformation

$$
u=\phi(x, y), \quad v=\psi(x, y) .
$$




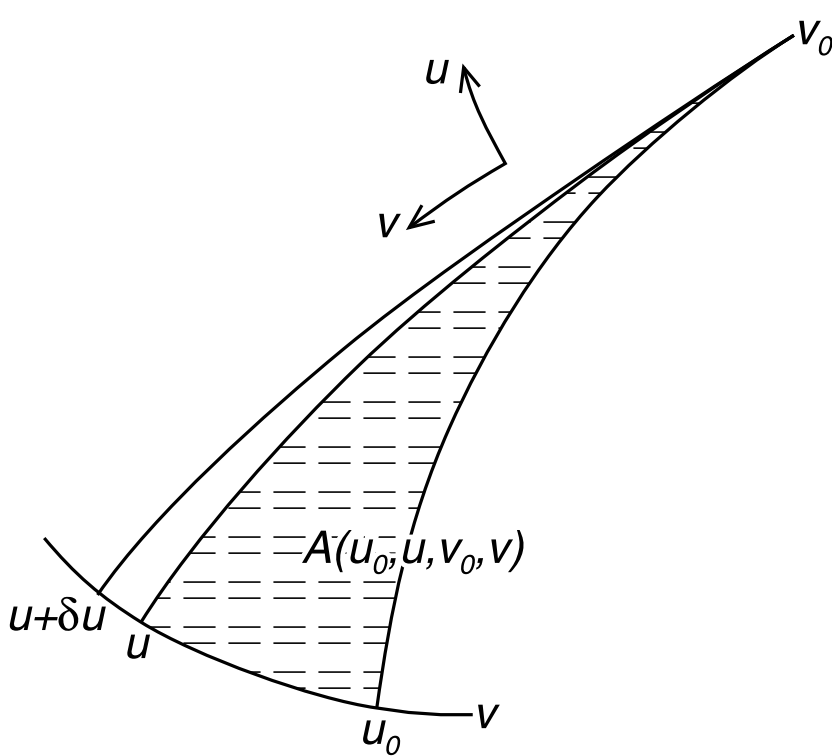

Figure 5. The curvilinear coordinate system $(u, v)$ and the stream tube used for the derivation.

Following Courant, a shorthand form of the derivatives of those functions is used for convenience:

$$
\begin{aligned}
& x_{u}=\frac{\partial}{\partial u} g(u, v) \\
& u_{x}=\frac{\partial}{\partial x} \varphi(x, y),
\end{aligned}
$$

and so on.

[25] In a curvilinear coordinate system the coordinates do not directly measure distances in space, and the conversion to distance is achieved using scaling factors $h^{u}$ and $h^{v}$ defined by (Jeffreys and Jeffreys [1956, section 4.14] with a change of notation)

$$
\begin{aligned}
& h^{u^{2}}=x_{u}^{2}+y_{u}^{2} \\
& h^{v^{2}}=x_{v}^{2}+y_{v}^{2} .
\end{aligned}
$$

[26] The partial derivatives must exist, and the scale factors must be nonzero throughout the domain of analysis. The network of slope lines and contours obeys this requirement so long as the surface is continuously differentiable, except where the surface is flat, i.e., at peaks, sinks, and saddles. As noted above, these requirements restrict the domain of analysis to areas away from peaks, sinks, and saddles and away from lines where derivatives are discontinuous, such as cliffs and sharp ridges and valleys.

[27] The quantity

$$
J=\left|\begin{array}{ll}
x_{u} & x_{v} \\
y_{u} & y_{v}
\end{array}\right|=x_{u} y_{v}-x_{v} y_{u}
$$

is known as the Jacobian and represents the infinitesimal area in the $(u, v)$ coordinate system, so the area of a region bounded by lines of constant $u$ and constant $v$ is [Jeffreys and Jeffreys, 1956, section 4.14]

$$
A=\iint J d u d v
$$

The right handedness of the $(u, v)$ system as defined here ensures that $J$ is always positive. In particular, for a stream tube bounded by streamlines at $u_{0}$ and $u$ extending from a hilltop at $v_{0}$ to a contour at $v$ as shown in Figure 5, the area is

$$
A\left(u_{0}, u, v_{0}, v\right)=\int_{u 0}^{u} \int_{v_{0}}^{v} J d p d q .
$$

The orthogonality of the $(u, v)$ coordinate system, arising from the fact that slope lines are always perpendicular to contour lines, has several consequences that will be useful in the following derivation [Jeffreys and Jeffreys, 1956, section 4.14, equation (7)]:

$$
x_{u} x_{v}+y_{u} y_{v}=0
$$

$$
J=h^{u} h^{v} .
$$

The latter result follows from the fact that the two sides of the infinitesimal area in the $(u, v)$ system can be considered to be straight lines of length $h^{u} d u$ and $h^{v} d v$ [Jeffreys and Jeffreys, 1956 , section 4.14], so the area is

$$
\begin{aligned}
d A & =h^{u} d u h^{v} d v \\
& =h^{u} h^{v} d u d v .
\end{aligned}
$$

We are interested in derivatives in the $u$ and $v$ directions in terms of distances rather than the $u$ and $v$ values themselves, so we will use $k$ and $l$ to denote distances in the $u$ and $v$ directions, respectively, and use the scale factors to express derivatives with respect to $u$ and $v$ :

$$
\frac{\partial}{\partial k}=\frac{1}{h^{u}} \frac{\partial}{\partial u}, \quad \frac{\partial}{\partial l}=\frac{1}{h^{v}} \frac{\partial}{\partial v} .
$$

With those definitions in place and considering the area of the stream tube between the lines $u$ and $u+\delta u$ in Figure 5, the definition of specific catchment area gives

$$
\begin{gathered}
a(u, v)=\lim _{\delta u \rightarrow 0} \frac{A\left(u_{0}, u+\delta u, v_{0}, v\right)-A\left(u_{0}, u, v_{0}, v\right)}{h^{u} \delta u}, \\
=\frac{1}{h^{u}} \frac{\partial A\left(u_{0}, u, v_{0}, v\right)}{\partial u}, \\
=\frac{1}{h^{u}} \frac{\partial}{\partial u} \int_{u_{0}}^{u} \int_{v_{0}}^{v} J d p d q \\
=\frac{1}{h^{u}} \int_{v_{0}}^{v} J d q .
\end{gathered}
$$


Note that this integral proceeds along a line of constant $u$ (a slope line) from a starting point $v_{0}$ which is located at the top of a hill to the point $(u, v)$.

[28] The rate of change of specific catchment area along the slope line is then given by

$$
\begin{aligned}
& \frac{\partial a}{\partial l}=\frac{1}{h^{v}} \frac{\partial a}{\partial v}, \\
& =\frac{1}{h^{v}} \frac{\partial}{\partial v}\left(\frac{1}{h^{u}} \int_{v_{0}}^{v} J d q\right) \\
& =\frac{1}{h^{v}}\left(\frac{1}{h^{u}} \frac{\partial}{\partial v} \int_{v_{0}}^{v} J d q+\int_{v_{0}}^{v} J d q \frac{\partial}{\partial v} \frac{1}{h^{u}}\right), \\
& =\frac{1}{h^{v}}\left(\frac{1}{h^{u}} J+h^{u} a \frac{\partial}{\partial v} \frac{1}{h^{u}}\right), \\
& =\frac{J}{h^{u} h^{v}}+\frac{h^{u}}{h^{v}} a \frac{\partial}{\partial v} \frac{1}{h^{u}}, \\
& =1+a \frac{h^{u}}{h^{v}}\left(\frac{-1}{h^{u^{2}}}\right) \frac{\partial h^{u}}{\partial v}, \\
& =1-a \frac{1}{h^{u} h^{v}} h_{v}^{u} .
\end{aligned}
$$

This is a similar form to (13) above, suggesting that the term $\frac{1}{h^{u} h^{v}} h_{v}^{u}$ plays the same role as plan (contour) curvature. The following shows they are in fact equivalent.

[29] Along a contour line where $v$ is constant the curvature is defined as [Courant, 1937, volume1, section 5.2.6]

$$
\kappa=\frac{x_{u} y_{u u}-y_{u} x_{u u}}{\left(x_{u}^{2}+y_{u}^{2}\right)^{3 / 2}},
$$

using the convention that curvature is positive for an angle with the $x$ axis that increases along the curve. The choice of positive contour curvature for diverging slope lines and $u$ directed toward the right looking downslope results in the opposite sign for curvature. With the change of sign and using (16) we have

$$
\kappa_{c}=\frac{y_{u} x_{u u}-x_{u} y_{u u}}{h^{u^{3}}} .
$$

Returning to the term in (34) that is expected to equal plan curvature, $\frac{1}{h^{u} h^{v}} h_{v}^{u}$, we first differentiate (16) with respect to $v$. This gives

$$
h_{v}^{u}=\frac{x_{u} x_{u v}+y_{u} y_{u v}}{h^{u}} .
$$

We also differentiate (21) with respect to $u$ :

$$
x_{u} x_{u v}+x_{v} x_{u u}+y_{u} y_{u v}+y_{v} y_{u u}=0 .
$$

Combining this with (37) gives

$$
h_{v}^{u}=\frac{-x_{v} x_{u u}-y_{v} y_{u u}}{h^{u}} .
$$

Returning to (16), we can write

$$
\frac{x_{u}^{2}}{h^{u^{2}}}+\frac{y_{u}^{2}}{h^{u^{2}}}=1
$$

which can be expressed as

$$
\begin{aligned}
& \frac{x_{u}}{h^{u}}=\cos A \\
& \frac{y_{u}}{h^{u}}=\sin A
\end{aligned}
$$

for some angle A. Similarly, from (17)

$$
\begin{aligned}
& \frac{x_{v}}{h^{v}}=\cos B \\
& \frac{y_{v}}{h^{v}}=\sin B .
\end{aligned}
$$

Dividing both (21) and (18) by $h^{u} h^{v}$ gives

$$
\begin{aligned}
& \cos A \cos B+\sin A \sin B=0 \\
& \cos A \sin B-\cos B \sin A=1 .
\end{aligned}
$$

Standard trigonometric identities transform these to

$$
\begin{aligned}
& \cos (B-A)=0 \\
& \sin (B-A)=1,
\end{aligned}
$$

and hence

$$
B-A=\frac{\pi}{2},
$$

leading to

$$
\begin{aligned}
& \cos B=-\sin A \\
& \sin B=\cos A .
\end{aligned}
$$

Thus,

$$
\begin{gathered}
\frac{x_{v}}{h^{v}}=-\frac{y_{u}}{h^{u}} \\
\frac{y_{v}}{h^{v}}=\frac{x_{u}}{h^{u}} .
\end{gathered}
$$

Substituting these identities into (39) gives

$$
h_{v}^{u}=\frac{h^{v} y_{u} x_{u u}-h^{v} x_{u} y_{u u}}{h^{u^{2}}}
$$

$$
\frac{1}{h^{u} h^{v}} h_{v}^{u}=\frac{y_{u} x_{u u}-x_{u} y_{u u}}{h^{u^{3}}}=\kappa_{c},
$$


confirming that in general

$$
\frac{\partial a}{\partial l}=1-a \kappa_{c}
$$

Note that the partial derivative is used here, unlike the simple case used to derive (13), where $a$ does not vary with position along the contour line.

[30] The only assumptions that have been made to arrive at this result are the existence of the first and second derivatives and the requirement that $h^{u}$ and $h^{v}$ are everywhere nonzero away from the hilltop, which is a requirement for a well-defined coordinate system.

[31] Equation (56) is the main result of this paper. It can be used to determine $a$ at any point by first constructing a single slope line upslope from the point of interest to a hilltop, then integrating (56) down that same slope line, with an initial condition of $a=0$ at the hilltop.

[32] The two terms of the right-hand side of (56) correspond to the two sources of change in specific catchment area: the constant term represents the source term, the increment to $a$ due to the increasing length of the slope line, while the second term represents the effect of divergence and convergence of the land surface. In divergent areas $\left(\kappa_{c}>0\right)$ the two terms compete and $a$ may increase or decrease along the slope line, while in convergent areas $\left(\kappa_{c}<0\right)$ the two terms combine to rapidly (exponentially) increase $a$.

[33] The source term, the constant 1 in (56), can be varied spatially for applications beyond the simple calculation of specific catchment area: if the Jacobian $J$, representing elemental area, is multiplied by a spatially varying source term in the area integral (20), that source term carries directly through to the final equation and replaces the constant 1 . This can be used, for example, to calculate surface runoff from spatially variable rainfall excess by setting the

source term to the rainfall excess.
[34] It is also worthwhile noting that $\frac{\partial a}{\partial l}$ is a topographic attribute in its own right that appears in Moore and Wilson's [1992] soil erosion and deposition index. Use of (56) rather than a numerical approximation to $\frac{\partial a}{\partial l}$ may improve the accuracy of the calculated erosion/deposition index.

[35] Equation (56) cannot, in general, be integrated analytically because the functional form of $\kappa_{c}(l)$ is unknown. For some simple surfaces the form of $\kappa_{c}(l)$ is known and the equation can be solved to give $a(l)$. In other cases a numerical solution is required.

\section{Analytic Solutions for Simple Cases}

[36] The simplest case for which an analytic solution to (56) is possible is the planar slope where $\kappa_{c}=0$ :

$$
\begin{gathered}
\frac{\partial a}{\partial l}=1 \\
a(l)=a_{0}+l,
\end{gathered}
$$

where $a_{0}$ is the specific catchment area at the top of the planar slope.

[37] Another simple case is the conical surface used to derive (13), where the radius of curvature $r_{c}$ is equal to flow length $l$ so $\kappa_{c}(l)=\frac{1}{l}$. The solution of (56) in this case is obtained by substituting $v=\frac{a}{l}$, which transforms (56) to

$$
\frac{d v}{d l}=\frac{1-2 v}{l}
$$

$$
\frac{d l}{l}=\frac{d v}{1-2 v}, \quad v \neq \frac{1}{2}
$$

Integration of (60) and expressing in terms of $a$ yields

$$
a=\frac{l}{2}-\frac{c}{l},
$$

which diverges as $l$ approaches 0 . The alternative solution of $v=\frac{1}{2}$ yields

$$
a(l)=\frac{l}{2}
$$

Like the planar slope, this equation can be readily derived from first principles.

[38] A more general form of this solution is where $\kappa_{c}(l)=$ $\frac{1}{c_{1} l+c_{0}}$, which yields

$$
a(l)=\frac{c_{0}+c_{1} l}{1+c_{1}}+\left(a_{0}-\frac{c_{0}}{1+c_{1}}\right)\left(\frac{c_{0}+c_{1} l}{c_{0}}\right)^{-1 / c_{1}},
$$

provided $c_{1} \neq-1$ and $c_{1} \neq 0$. Note that this form of $\kappa_{c}$ is not applicable where plan curvature changes sign, nor is it applicable when $\kappa_{c}(0)=0$ because $c_{0}$ would then be undefined. Note that

$$
\frac{c_{0}+c_{1} l}{c_{0}}=\frac{\kappa_{c}(0)}{\kappa_{c}(l)}
$$

which is a positive quantity provided that $\kappa_{c}$ does not change sign; this is important because the ratio is raised to a noninteger power. Equation (63) includes the solution to the convergent cone case with an outer radius of $R$. In this case $a_{0}=0$ and $\kappa_{c}(l)=\frac{1}{l-R}$, so $c_{1}=1$ and $c_{0}=-R$, which results in

$$
a(l)=\frac{l(2 R-l)}{2(R-l)}
$$

which again matches the solution from first principles.

[39] The two excluded cases in (63), $c_{1}=-1$ and $c_{1}=0$, give different solutions. For $c_{1}=-1$

$$
a(l)=\left(c_{0}-l\right)\left(\frac{a(0)}{c_{0}}-\log \left(\frac{c_{0}-l}{c_{0}}\right)\right) .
$$

The ratio $\frac{c_{0}-l}{c_{0}}$ is again equivalent to $\frac{\kappa_{c}(0)}{\kappa_{c}(l)}$.

[40] In the final case where $c_{1}=0, \kappa_{c}(l)$ is constant and nonzero and the solution to (56) is

$$
a(l)=\frac{1}{\kappa_{c}}-\left(\frac{1}{\kappa_{c}}-a_{0}\right) e^{-\kappa_{c} l},
$$

where $a_{0}$ is the specific catchment area at the top of the region with constant $\kappa_{c}$. When $\kappa_{c}$ is negative (a uniformly 
curved valley), the terrain is convergent and $a$ increases exponentially. When $\kappa_{c}$ is positive (a uniformly curved ridge), the terrain is divergent and the exponential term tends toward 0 with increasing $l$, so $a$ tends exponentially toward $\frac{1}{\kappa_{c}}=r_{c}$, the radius of curvature of the contour lines. When $a=\frac{\kappa_{c}}{\kappa_{c}}$ the two opposing terms in (56), divergence and accumulation of area, balance exactly, so $\frac{\partial a}{\partial l}=0$.

[41] The analytical solutions on mathematical surfaces are not the main strength of equation (56). In fact, most of the above results are easier to derive directly from equation (1) than from (56). The main value of (56) is as a rigorous foundation for exploring the behavior of specific catchment area along a slope line on arbitrary surfaces. It provides an equation that can be numerically integrated along a slope line in complex topography to yield an accurate value of specific catchment area that can be used to assess approximate numerical methods.

\section{Numerical Methods}

[42] To apply equation (56) on real topography, a mathematical representation of the real surface is required. This involves several steps with various choices of how the elevations of the real surface are measured and how those measurements are represented and converted to a mathematical form. The traditional approach has been to measure the land surface photogrammetrically, represent the heights as a contour map, and derive a grid of elevations from the contours using a suitable interpolation technique [e.g., Hutchinson and Gallant, 2000]. A suitable mathematical surface can then be derived from the grid. More recently, surface elevations are being acquired as a dense set of measured spot heights by laser altimetry or GPS surveying. These are interpolated to a grid without using contours as an intermediate representation. The grid of elevations can also be derived directly from stereo image pairs using soft photogrammetry or from radar interferometry.

[43] There are also choices in constructing a mathematical surface from the grid DEM. To apply equation (56), the slope lines must be defined by continuous first derivatives and the plan curvature, which depends on second derivatives of the land surface, must be integrable. As indicated in section 1 , this is most easily ensured by having a surface with continuous first derivatives and piecewise continuous second derivatives. This prevents slope lines from merging, which would violate the assumptions underlying the curvilinear coordinate system. Accordingly, each slope line starts at a hilltop and continues to a sink or the coast but in practice typically stops at the edge of the DEM.

[44] In this paper we derive the mathematical surface from a grid DEM using biquadratic spline interpolation [de Boor, 1978]. Biquadratic splines are tensor products of univariate quadratic splines, which have excellent approximation properties, superior to those of cubic splines when applied to general continuous functions [Marsden, 1974]. The biquadratic spline interpolation method provides a surface with the required continuous first derivatives and piecewise continuous second derivatives. Using this surface, slope lines are constructed of many short straight lines with a length that adapts to the complexity of the surface so that small steps are used where the slope line curves tightly.
Stationary points where the surface is exactly flat, namely, hilltops, saddles, and sinks, are recognized by the numerical scheme, and flow lines terminate at hilltops on the uphill end and on sinks at the downhill end or when they continue to the edge of the DEM. Spurious stationary points can be generated by smooth interpolation methods, but this problem was not encountered in the experiments shown in this paper, largely because of the sink-free nature of the DEM produced by ANUDEM and the high relief of the landscape used for the experiments.

[45] The differential equation (56) gives an exact solution of $a$ for the chosen mathematical surface, however that is defined. Approximations are made in obtaining the mathematical surface from the real terrain surface and in the numerical solution of (56), but the approximations due to the numerical solution can be made negligibly small by suitable numerical methods. The derived values of $a$ will thus not exactly represent the specific catchment area of the real land surface, but that is due only to the approximations in the representation of the real surface as a grid DEM and the conversion of the grid to a mathematical surface.

[46] Numerical integration of (56) along a slope line consists of computing the value of $a$ at the end of each successive line segment, starting from 0 at the top of the slope line, using a model of $\kappa_{c}$ along the line segment. The value of $\kappa_{c}$ is readily calculated at the endpoints of each line segment from the derivatives of the interpolated biquadratic spline surface:

$$
\begin{gathered}
\kappa_{c}=\frac{-z_{x x} z_{y}^{2}+2 z_{x y} z_{x} z_{y}-z_{y y} z_{x}^{2}}{\left(z_{x}^{2}+z_{y}^{2}\right)^{3 / 2}} \\
z_{x}=\frac{\partial z}{d x}, z_{x y}=\frac{\partial^{2} z}{\partial x \partial y}, \text { etc. }
\end{gathered}
$$

The simplest approximation to $\kappa_{c}(l)$ along the line segment is to use a constant $\kappa_{c}$ equal to the average of the endpoint values and apply equation (67) to compute the new value of $a$ at the end of the line. A more accurate approach is to use (63) with $c_{1}$ and $c_{0}$ calculated from $\kappa_{c}$ at the beginning and end of the line segment, provided that the conditions on that equation are met. The algorithm applied here to demonstrate the method uses (63) except where $c_{1}$ is close to 0 or 1 or $\kappa_{c}$ changes sign along the line segment; in any of these cases (67) is used.

[47] Two further numerical details need to be considered when using (67). First, if $\kappa_{c} l$ is quite small, the exponential term is very close to 1 and loss of precision is likely due to the subtraction. In this case, the planar slope solution (58) can be used. In the implementation used here, (58) was used whenever the absolute value of $\kappa_{c} l$ was less than $10^{-4}$.

[48] Second, if $\kappa_{c} l$ is quite large, the exponential calculation can underflow. This is not a problem from a mathematical point of view, but underflow may cause an unwanted floating point exception, so the solution

$$
a(l)=\frac{1}{\kappa_{c}}
$$

could be used where $\kappa_{c} l$ exceeds about 20. The implementation used here did not use this adjustment.

[49] Finally, where a slope line runs along a valley bottom in convergent terrain, $a$ will increase exponentially, even- 


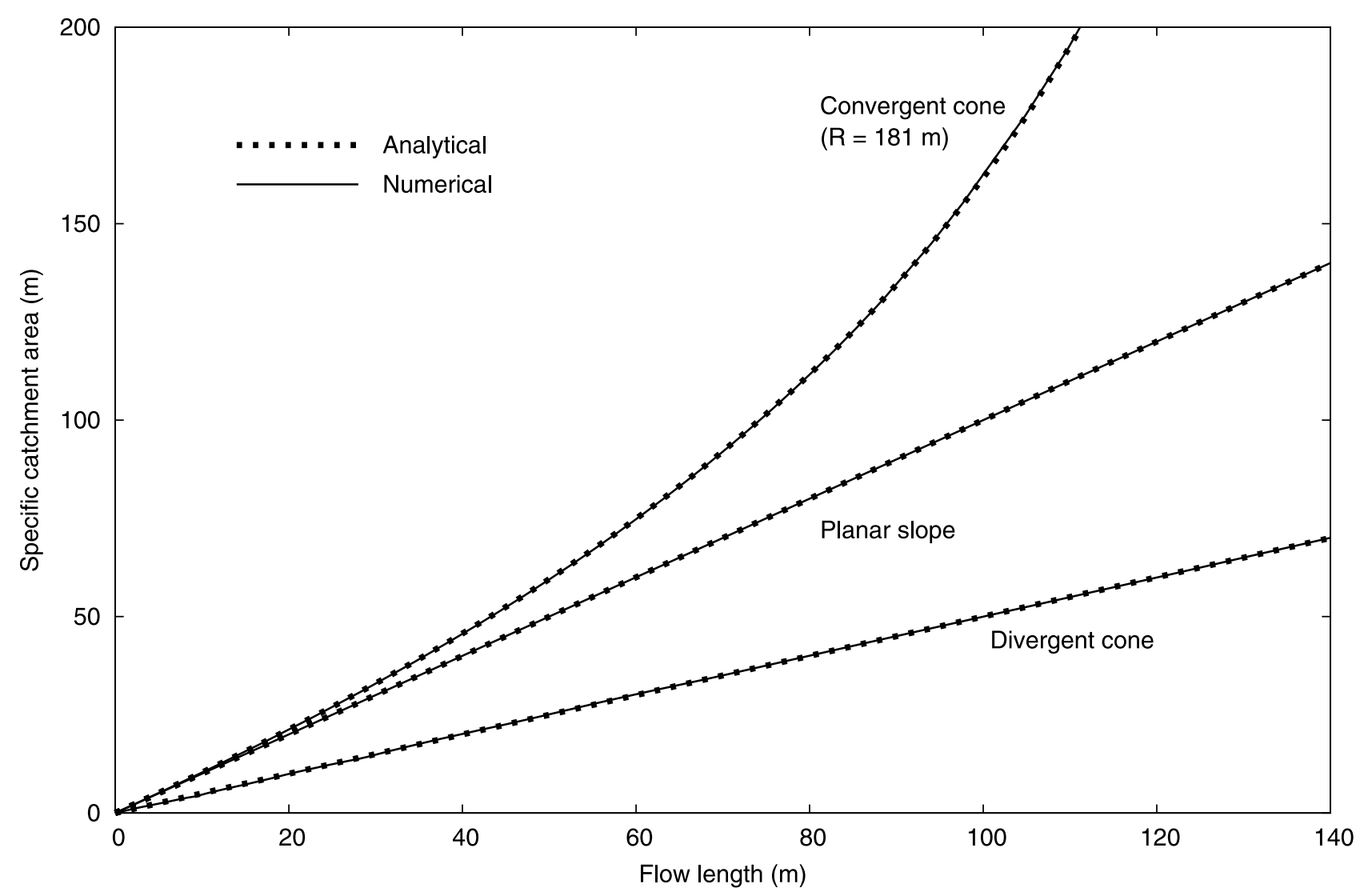

Figure 6. Comparison of analytical solutions for $a$ with values computed numerically on $10 \mathrm{~m}$ resolution DEMs of a planar slope, divergent cone, and convergent cone. The analytical and numerical solutions are indistinguishable.

tually causing numerical overflow. The implementation used here ceased integrating (56) once $a$ exceeds $10^{50}$. The value of $a$ loses any physical meaning at a value much less than this.

[50] Computing specific catchment area for every cell in a DEM would require constructing enough slope lines across the surface that a slope line passes near the center of each cell. Equation (56) could then be integrated along each line, storing results in cells along the way. This complete coverage has not been attempted but would require much more computation than any of the standard contributing area methods. The calculations would be further complicated by the spurious flat points inevitably created by a continuous interpolation. For these reasons, this method is viewed as a precise reference against which other methods can be compared rather than an operational tool for computing specific catchment area.

\section{Numerical Results}

\subsection{Simple Surfaces With Analytic Solutions}

[51] Figure 6 shows specific catchment area computed using equation (56) for three simple surfaces commonly used for testing grid-based specific catchment area and contributing area methods. The three surfaces are an inclined plane, a divergent cone, and a convergent cone, each constructed as a $10 \mathrm{~m}$ resolution grid. For each surface the numerical solution was obtained using the above algo- rithm, and the analytical solution was obtained using (58) for the inclined plane, (62) for the divergent cone, and (65) for the convergent cone. The results show that the numerical and analytical solutions are virtually identical, indicating a well-controlled numerical integration.

\subsection{Comparison With the Explicit Stream Tube}

[52] Figure 7 shows specific catchment area computed by numerical integration of (56), compared with specific catchment area and flow width computed using the stream tube method shown in Figure 3. There is very close agreement between the two estimates of $a$ in the areas where the width of the stream tube is well defined. On the ridge area (about the first $200 \mathrm{~m}$ of the slope line) where the stream tube width is unstable, the differential equation-based method is clearly stable and consistent with the expected behavior in divergent areas. As indicated by equation (67), specific catchment area tends to the radius of curvature along uniform divergent flow paths. The $20 \mathrm{~m}$ resolution of the DEM used here combined with the smooth biquadratic spline interpolation results in a radius of curvature of approximately $10 \mathrm{~m}$ for contours on sharp ridgelines and hence a specific catchment area of about $10 \mathrm{~m}$ for the ridgeline flow path.

[53] From the point where the stream tube begins to contract $(l>500 \mathrm{~m})$, the specific catchment area computed from (56) increases exponentially. Unlike the values obtained from the stream tube, this exponential increase continues 


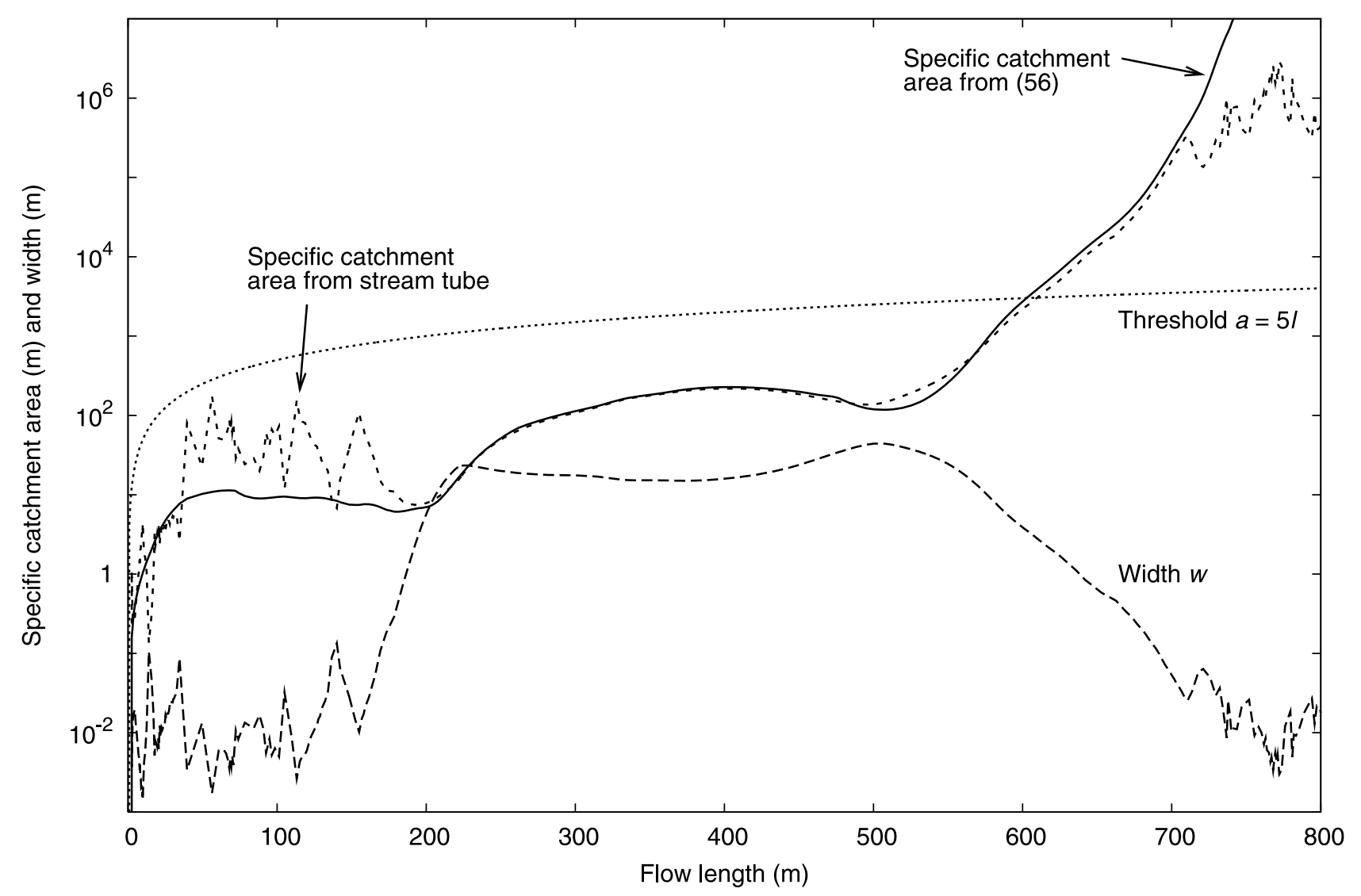

Figure 7. Comparison of $a$ computed from equation (56) with $a$ computed from the stream tube of Figure 2. The flow width $w$ from Figure 4 is also shown. The line $a=5 l$ is one possible threshold for identifying the onset of convergent flow in this landscape.

through the entire convergent section. The exponential increase is due to the large negative plan curvature in the bottom of the valley. As noted in section 1, the connection between water flow and specific catchment area based upon slope lines is lost in conditions of concentrated flow and there is little value in continuing the calculation of specific catchment area in these areas. A suitable condition for stopping the integration of specific catchment area needs to be developed and might be related to geomorphic thresholds linked to channel initiation. As an example, the line $a=5 l$ in Figure 7 illustrates one possible threshold that may be suitable for this landscape.

\subsection{Comparison With Conventional Methods}

[54] The most useful application of (56) is as a standard against which the more efficient approximate algorithms such as D8, M8, DEMON, and D $\infty$ can be compared in complex landscapes. Contributing area was calculated for the DEM using the D8, M8, and DEMON methods by the TAPES-G program [Gallant and Wilson, 1996; Gallant and Wilson, 2000] and using the D $\infty$ method [Tarboton, 1997] by the Taudem tool version 3.1 for Esris ArcGIS 9 (http:// hydrology.usu.edu/taudem/taudem5.0/index.html). Conversion of contributing area $A$ to specific catchment area $a$ requires a flow width, and conventional methods were applied here. For the D8 and M8 methods flow width was the cell size $h$ for cardinal D8 flow directions and $\sqrt{2} h$ for diagonal D8 flow directions. For the DEMON method flow width is $(|\sin \psi|+|\cos \psi|) h$, where $\psi$ is the aspect angle [Costa-Cabral and Burges, 1994, equation (7)]. The Taudem calculation of specific catchment area using the D $\infty$ method assumes that flow width is equal to grid size $h$ regardless of flow direction.

[55] Figure 8 shows values of specific catchment area extracted from points along the central slope line of Figure 2 for the four methods and the results of (56) for comparison (note that the vertical axis scale is expanded compared to Figure 7). The behavior of the methods is different in the four different regions of the flow path: the ridge section from 0 to $220 \mathrm{~m}$ flow length, the planar hillslope section from 220 to $400 \mathrm{~m}$, the divergent hillslope from 400 to $500 \mathrm{~m}$, and the convergent section beyond $500 \mathrm{~m}$.

[56] Along the ridge section all the grid-based methods overestimate the specific catchment area compared to (56). This is largely due to the incorrect estimation of flow width in strongly diverging terrain. Flow should be able to leave the cell over most of the cell boundary, resulting in a flow width 2-3 times larger than the cell size $h$. The irregularity in the values for D8 and M8 is due to the flow width changing abruptly from $h$ to $\sqrt{2} h$ as the flow direction changes between cardinal and diagonal. The D $\infty$ method does not vary flow width with flow direction, so it does not exhibit any irregularity, while DEMON method varies flow width gradually with aspect and thus shows a more subdued variation. The M8 method gives the largest values in this section presumably due to some accumulation of flow along the ridge by the multiple-flow direction algorithm. Both 


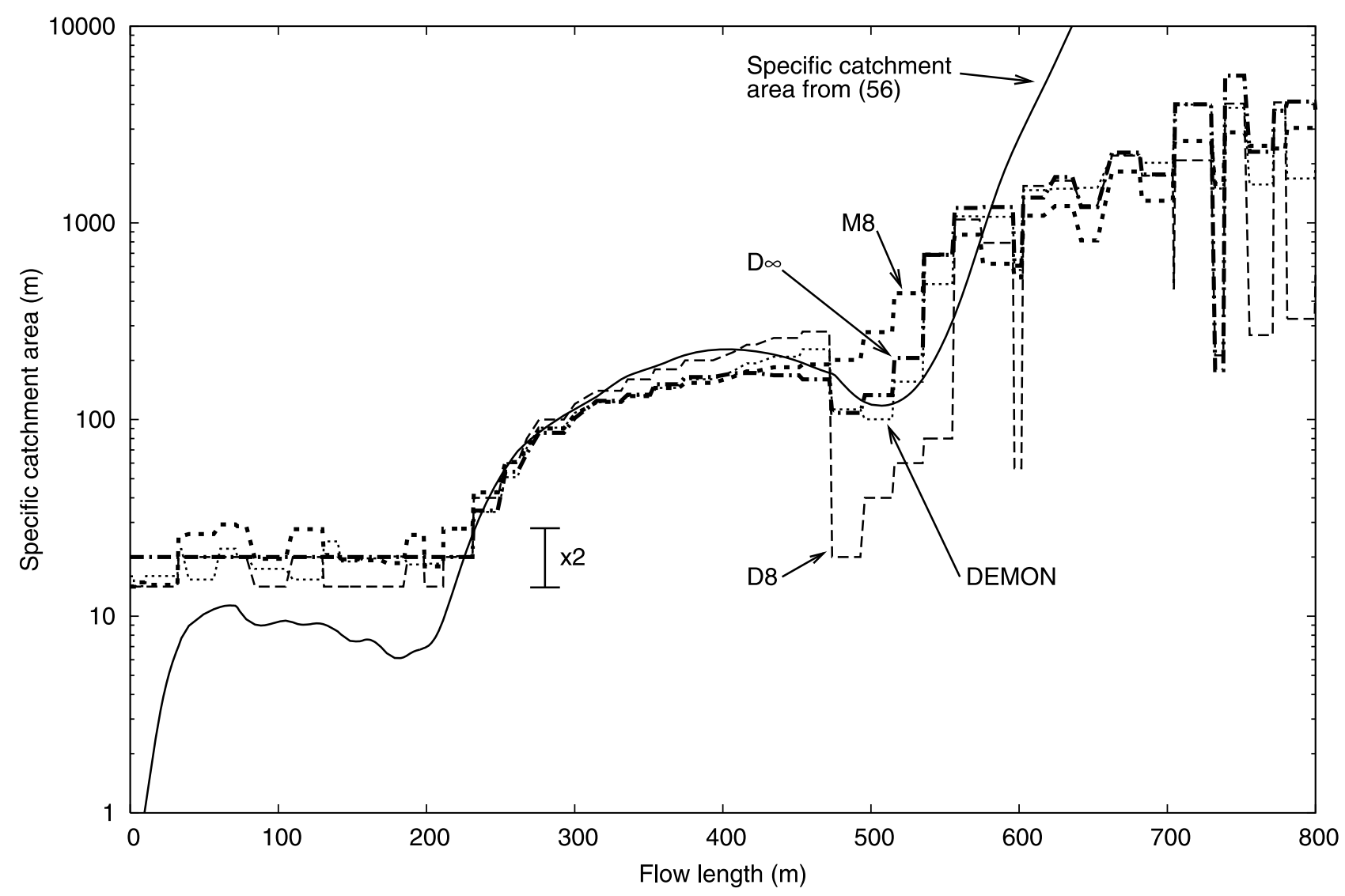

Figure 8. Comparison of $a$ computed from equation (56) with $a$ estimated using established cell-based methods for the central slope line of Figure 2 . The bar labeled $\times 2$ shows a ratio of 2 on the logarithmic scale of the vertical axis.

the D8 and D $\infty$ methods give single-cell contributing areas $\left(400 \mathrm{~m}^{2}\right)$, and hence specific catchment areas around $20 \mathrm{~m}$, along the entire ridge.

[57] Along the planar hillslope section all the methods show a similar pattern of specific catchment area but with some differences in bias. The D8 method appears to be the most accurate in this section; the easterly orientation of the slope line suits the simple D8 method of flow accumulation in one of the eight directions, and similarly accurate results would not be expected for noncardinal flow orientations [Costa-Cabral and Burges, 1994]. DEMON, M8, and D $\infty$ behave very similarly and all tend to underestimate to an increasing degree down the hillslope, reaching about $20 \%$ at $400 \mathrm{~m}$ flow length.

[58] The behaviors diverge markedly on the gently divergent hillslope from 400 to $500 \mathrm{~m}$. The D8 algorithm predicts increasing specific catchment area until it reaches one cell at a flow length of $470 \mathrm{~m}$, as that is treated as a ridge with no inflow, and specific catchment area drops back to $20 \mathrm{~m}$. It continues underestimating specific catchment area for the remainder of this section. This is a substantial overreaction to the modest divergence in that area. The M8 algorithm barely responds to the divergence, and its estimate of specific catchment area does not decrease anywhere in this section. DEMON's estimate increases gradually in the first part of this section, then drops abruptly to a level consistent with the results from (56). D $\infty$ behaves most similarly to (56), decreasing gradually in the first part of the divergent section, then dropping more abruptly in the most divergent area. In the transition from divergent to convergent around 520-560 m, M8, DEMON, and D $\infty$ all overestimate specific catchment area.

[59] In the convergent section the solution to (56) no longer corresponds to the ratio of area to width at the scale of a DEM cell, so the estimates from the cell-based methods differ significantly from the analytic solution. This is also the regime where assuming flow width is equal to cell size is suspect: most of the flow is confined to a channel which may have a width quite different from the cell size, and the flow width varies with water depth.

[60] The specific catchment areas derived by the four methods show a generally increasing trend as expected in a channel. The abrupt drops occur where the slope line derived from the interpolated surface crosses a cell that is not considered to be part of the channel by the grid-based methods. Note that these drops are smallest for the M8 method because of its dispersion of flow across more than one cell in the valley bottom, but the average value for M8 is lower for the same reason. The D $\infty$ and D8 values closely match over most of the channel section.

[61] A more thorough comparison of the four methods with (56), and an extension of the analysis to include more recently developed methods such as D8-LTD and $\mathrm{MD} \propto$, would be appropriate to examine their strengths and weaknesses in complex terrain. The limited analysis carried out here suggests that the $\mathrm{D} \infty$ method responds best to variations in surface shape on hillslopes. All methods overestimate $a$ in strongly divergent terrain, suggesting that a 
revision of the methods for estimating $w$, and perhaps $A$, in these areas is desirable. It should also be noted that, as pointed out by Orlandini and Moretti [2009], it is possible for a cell-based method to obtain correct values of $a$ or $A$ while incorrectly representing the flow paths within that contributing area, and this analysis does not address the validity of the flow paths used by the cell-based methods.

[62] It is not yet clear whether the discrepancies between the approximate cell-based methods and the analytical solution have any impact on the representation of physical processes using the approximate specific catchment area. It may be that the errors that arise from the approximations involved in measuring surface heights and creating a grid DEM are larger than the errors that arise directly from the estimation of specific catchment area from the resulting grid. The method described here provides the opportunity to make these comparisons and determine whether better methods for calculating specific catchment area are needed.

\subsection{Application to Dispersal Areas}

[63] While contributing area $A$ and specific catchment area $a$ are the most commonly used area attributes, dispersal area $D$ and specific dispersal area $d$ are also used in some circumstances. Dispersal area is the area downslope of a contour segment and defines the area to which water drains from the contour segment rather than the area supplying water to the contour segment. Dispersal area can play a role in predicting patterns of soil water content [Speight, 1980] and in predicting soil properties [McKenzie and Ryan, 1999]. Delineating dispersal areas is also valuable for predicting the extent of contamination from a pollutant source area [CostaCabral and Burges, 1994].

[64] Dispersal area and specific dispersal area obey identical rules to contributing area and specific catchment area, except that slope lines are constructed downslope from the location of interest rather than upslope. The methods described in this paper are equally applicable to calculation of dispersal areas, apart from a change of sign due to the decrease in dispersal area when proceeding downslope. The dispersal area for a stream tube bounded by streamlines at $u_{0}$ and $u$ extending from contour at $v$ to a flow termination point at $v_{1}$ is

$$
D\left(u_{0}, u, v, v_{1}\right)=\int_{u 0}^{u} \int_{v}^{v_{1}} J d p d q
$$

so specific dispersal area is

$$
d=\frac{1}{h^{u}} \int_{v}^{v_{1}} J d q .
$$

The rate of change of specific dispersal area along the slope line is

$$
\begin{gathered}
\frac{\partial d}{\partial l}=\frac{1}{h^{v}} \frac{\partial d}{\partial v} \\
=\frac{1}{h^{v}} \frac{\partial}{\partial v}\left(\frac{1}{h^{u}} \int_{v}^{v_{1}} J d q\right),
\end{gathered}
$$

$$
\begin{gathered}
=\frac{1}{h^{v}}\left(\frac{1}{h^{u}} \frac{\partial}{\partial v} \int_{v}^{v_{1}} J d q+\int_{v}^{v_{1}} J d q \frac{\partial}{\partial v} \frac{1}{h^{u}}\right), \\
=\frac{1}{h^{v}}\left(\frac{1}{h^{u}}(-J)+h^{u} d \frac{\partial}{\partial v} \frac{1}{h^{u}}\right) \\
=-1-d \frac{1}{h^{u} h^{v}} h_{v}^{u} \\
=-1-d \kappa_{c} .
\end{gathered}
$$

The integration of (77) must commence at the downslope termination of the slope line with $d=0$ and proceed upslope in the opposite sense to $l$, so the quantity to integrate in the upslope direction is $1+\kappa_{c} d$. Finding a suitable downslope termination point is more difficult than for the specific catchment area case: sinks in high-relief terrain are rare compared to peaks, and most good quality DEMs aim to eliminate sinks in such areas to avoid impediments to downhill flow. Slope lines will typically continue downslope to a shoreline, or the edge of the DEM, leading to a lot of wasted work integrating (77) in long convergent valleys where $d$ remains close to 0 . A more sophisticated criterion for stopping the downslope construction of flow lines would be useful, such as entry to a channel or strongly convergent terrain, and an approach similar to the criterion for calculating an effective path length suggested above may be suitable.

\section{Conclusion}

[65] The nonlinear differential equation (56) defines the rate of change of specific catchment area along a slope line emanating from a hilltop as a function of specific catchment area and plan curvature at every point on the slope line. Integrating the equation along the slope line from the hilltop yields specific catchment area at every point along the line without having to estimate areas and flow widths, as all existing grid- and contour-based estimation methods do. Analytical solutions are possible in simple cases, but in general, numerical methods must be used.

[66] The equation and the methods for its numerical solution presented here provide a precise reference against which approximate methods can be tested on real topographic surfaces rather than just on simple mathematical surfaces such as planes and cones. A preliminary evaluation of the four commonly used methods supports previous analyses that have shown that the more sophisticated methods (M8, DEMON, and D $\infty$ ) perform well on hillslopes, with the D8 method giving poor results. D $\infty$ appears to be the most accurate method overall. On ridges and hilltops, all four methods overestimate specific catchment area, apparently because of underestimation of flow width.

[67] Acknowledgments. The authors thank Kit Rutherford and Warrick Dawes for constructive reviews of the manuscript before submission. We are indebted to David Tarboton and Stefano Orlandini for their thoughtful and instructive reviews that helped shape this paper. Those reviews prompted the conceptual separation between topographic slope lines and water flow lines, the discussions on continuity, and the several 
steps between real topography and the mathematical surface used in this analysis. We particularly thank Stefano Orlandini for suggesting the direction for a more rigorous mathematical derivation than our original attempt.

\section{References}

Beven, K. J., and M. J. Kirkby (1979), A physically based variable contributing area model of basin hydrology, Hydrol. Sci. Bull., 24, 43-69, doi:10.1080/02626667909491834.

Cayley, A. (1859), On contour and slope lines, London Edinburgh Dublin Philos. Mag. J. Sci., Ser. 4, 18, 264-268.

Chirico, G. B., A. W. Western, R. B. Grayson, and G. Blöschl (2005), On the definition of flow width for calculating specific catchment area patterns from gridded elevation data, Hydrol. Processes, 19(13), 2539-2556, doi:10.1002/hyp.5730.

Costa-Cabral, M. C., and S. J. Burges (1994), Digital elevation model networks (DEMON): A model of flow over hillslopes for computation of contributing and dispersal areas, Water Resour. Res., 30(6), 1681-1692, doi:10.1029/93WR03512.

Courant, R. (1937), Differential and Integral Calculus, Blackie, London.

Dawes, W. R., and D. Short (1994), The significance of topology for modeling the surface hydrology of fluvial landscapes, Water Resour. Res., 30(4), 1045-1055, doi:10.1029/93WR02479.

de Boor, C. (1978), A Practical Guide to Splines, Springer, Berlin.

Dietrich, W. E., C. J. Wilson, D. R. Montgomery, and J. McKean (1993), Analysis of erosion thresholds, channel networks and landscape morphology using a digital terrain model, J. Geol., 101(2), 259-278, doi:10.1086/648220.

Fairfield, J., and P. Leymarie (1991), Drainage networks from grid digital elevation models, Water Resour. Res., 27(5), 709-717, doi:10.1029/ 90WR02658.

Freeman, T. G. (1991), Calculating catchment area with divergent flow based on a regular grid, Comput. Geosci., 17(3), 413-422, doi:10. 1016/0098-3004(91)90048-I.

Gallant, J. C., and J. P. Wilson (1996), TAPES-G: A grid-based terrain analysis program for the environmental sciences, Comput. Geosci., 22(7), 713-722, doi:10.1016/0098-3004(96)00002-7.

Gallant, J. C., and J. P. Wilson (2000), Primary topographic attributes, in Terrain Analysis: Principles and Applications, edited by J. P. Wilson and J. C. Gallant, pp. 51-85, John Wiley, New York.

Gessler, P. E., I. D. Moore, N. J. McKenzie, and P. J. Ryan (1995), Soillandscape modeling and spatial prediction of soil attributes, Int. J. Geogr. Inf. Syst., 9, 421-432, doi:10.1080/02693799508902047.

Gruber, S., and S. Peckham (2009), Land surface parameters and objects in hydrology, in Geomorphometry: Concepts, Software, Applications, edited by T. Hengl and H. I. Reuter, pp. 171-194, Elsevier, Amsterdam, doi:10.1016/S0166-2481(08)00007-X.

Güntner, A., J. Seibert, and S. Uhlenbrook (2004), Modeling spatial patterns of saturated areas: An evaluation of different terrain indices, Water Resour. Res., 40, W05114, doi:10.1029/2003WR002864.

Hutchinson, M. F. (1988), Calculation of hydrologically sound digital elevation models, paper presented at Third International Symposium on Spatial Data Handling, Int. Geogr. Union, Sydney, N. S. W., Australia, 17-19 Aug.

Hutchinson, M. F. (1989), A new procedure for gridding elevation and stream line data with automatic removal of spurious pits, J. Hydrol., 106, 211-232, doi:10.1016/0022-1694(89)90073-5.

Hutchinson, M. F. (2006) ANUDEM version 5.2, Cent. for Resour. and Environ. Stud., Aust. Natl. Univ., Canberra. (Available at http:// fennerschool.anu.edu.au/publications/software/anudem.php.)

Hutchinson, M. F., and J. C. Gallant (2000), Digital elevation models and representation of terrain shape, in Terrain Analysis: Principles and Applications, edited by J. P. Wilson and J. C. Gallant, pp. 29-49, John Wiley, New York.

Jeffreys, H., and B. Jeffreys (1956), Methods of Mathematical Physics, 3rd ed., Cambridge Univ. Press, Cambridge, U. K.

Lea, N. J. (1992), An aspect driven kinematic routing algorithm, in Overland Flow: Hydraulics and Erosion Mechanics, edited by A. J. Parsons and A. D. Abrahams, pp. 393-407, Chapman and Hall, New York.

Mackey, B. G., I. C. Mullen, K. A. Baldwin, J. C. Gallant, R. A. Sims, and D. W. McKenney (2000), Towards a spatial model of boreal forest ecosystems: The role of digital terrain analysis, in Terrain Analysis: Principles and Applications, edited by J. P. Wilson and J. C. Gallant, pp. 391-422, John Wiley, New York.

Marsden, M. J. (1974), Quadratic spline interpolation, Bull. Am. Math. Soc., 80(5), 903-907, doi:10.1090/S0002-9904-1974-13566-4.
Maxwell, J. C. (1870), On hills and dales, London Edinburgh Dublin Philos. Mag. J. Sci., Ser. 4, 40, 421-427.

McKenzie, N. J., and P. J. Ryan (1999), Spatial prediction of soil properties using environmental correlation, Geoderma, 89(1-2), 67-94, doi:10. 1016/S0016-7061(98)00137-2.

McKenzie, N. J., P. E. Gessler, P. J. Ryan, and D. A. O’Connell (2000), The role of terrain analysis in soil mapping, in Terrain Analysis: Principles and Applications, edited by J. P. Wilson and J. C. Gallant, pp. 245-265, John Wiley, New York.

McNamara, J. P., A. D. Ziegler, S. H. Wood, and J. B. Vogler (2006), Channel head locations with respect to geomorphologic thresholds derived from a digital elevation model: A case study in northern Thailand, For. Ecol. Manage., 224(1-2), 147-156, doi:10.1016/j.foreco. 2005.12.014.

Mishra, S. K., R. Sarkar, S. Dutta, and S. Panigrahy (2008), A physically based hydrological model for paddy agriculture dominated hilly watersheds in tropical region, J. Hydrol., 357, 389-404, doi:10.1016/j.jhydrol. 2008.05.019.

Moore, I. D., and G. R. Burch (1986), Sediment transport capacity of sheet and rill flow: Application of unit stream power theory, Water Resour. Res., 22(8), 1350-1360, doi:10.1029/WR022i008p01350.

Moore, I. D., and R. B. Grayson (1991), Terrain-based catchment partitioning and runoff prediction using vector elevation data, Water Resour. Res., 27(6), 1177-1191, doi:10.1029/91WR00090.

Moore, I. D., and J. P. Wilson (1992), Length-slope factors for the revised universal soil loss equation: Simplified method of estimation, J. Soil Water Conserv., 47(5), 423-428.

Moore, I. D., T. W. Norton, and J. E. Williams (1993), Modelling environmental heterogeneity in forested landscapes, J. Hydrol., 150, 717-747, doi:10.1016/0022-1694(93)90133-T.

Moretti, G., and S. Orlandini (2008), Automatic delineation of drainage basins from contour elevation data using skeleton construction techniques, Water Resour. Res., 44, W05403, doi:10.1029/2007WR006309.

O'Callaghan, J. F., and D. M. Mark (1984), The extraction of drainage networks from digital elevation data, Comput. Vision Graphics Image Process., 28, 323-344, doi:10.1016/S0734-189X(84)80011-0.

O'Loughlin, E. M. (1986), Prediction of surface saturation zones on natural catchments by topographic analysis, Water Resour. Res., 22(5), 794-804, doi:10.1029/WR022i005p00794.

Onstad, C. A., and D. L. Brakensiek (1968), Watershed simulation by the stream path analogy, Water Resour. Res., 4(5), 965-971, doi:10.1029/ WR004i005p00965.

Orlandini, S., and G. Moretti (2009), Determination of surface flow paths from gridded elevation data, Water Resour. Res., 45, W03417, doi:10. 1029/2008WR007099.

Orlandini, S., G. Moretti, M. Franchini, B. Aldighieri, and B. Testa (2003), Path-based methods for the determination of nondispersive drainage directions in grid-based digital elevation models, Water Resour. Res., 39(6), 1144, doi:10.1029/2002WR001639.

Quinn, P., K. Beven, P. Chevallier, and O. Planchon (1991), The prediction of hillslope flow paths for distributed hydrologic modelling using digital terrain models, Hydrol. Processes, 5, 59-79, doi:10.1002/hyp. 3360050106.

Quinn, P. F., K. J. Beven, and R. Lamb (1995), The $\ln (a / \tan \beta)$ index: How to calculate it and how to use it within the TOPMODEL framework, Hydrol. Processes, 9, 161-182, doi:10.1002/hyp.3360090204.

Seibert, J., and B. L. McGlynn (2007), A new triangular multiple flow direction algorithm for computing upslope areas from gridded digital elevation models, Water Resour. Res., 43, W04501, doi:10.1029/ 2006WR005128.

Speight, J. G. (1980), The role of topography in controlling throughflow generation: A discussion, Earth Surf. Processes, 5, 187-191, doi:10. 1002/esp.3760050209.

Tarboton, D. G. (1997), A new method for the determination of flow directions and upslope areas in grid digital elevation models, Water Resour. Res., 33(2), 309-319, doi:10.1029/96WR03137.

Ventura, S. J., and B. J. Irvin (2000), Automated landform classification methods for soil-landscape studies, in Terrain Analysis: Principles and Applications, edited by J. P. Wilson and J. C. Gallant, pp. 267-294, John Wiley, New York.

J. C. Gallant, CSIRO Land and Water, GPO Box 1666, Canberra, ACT 2601, Australia. (john.gallant@csiro.au)

M. F. Hutchinson, Fenner School of Environment and Society, Australian National University, Canberra, ACT 0200, Australia. 